\title{
PERSISTENCE OF THE CIRCULATION IN THE POSTERIOR VASCULAR SHEATH OF THE \\ LENS IN THE RIGHT EYE OF A GIRL AGED FOURTEEN YEARS
}

\author{
BY
}

\section{HARRIS TOMKIN}

DUBLIN

I SHOWED this case at the recent Meeting of the Irish Ophthalmological Society.

This patient attended the Out-Patient Department of the Royal Victoria Eye and Ear Hospital, Dublin, five years ago, and the condition has undergone no change.

My rough notes, at that time, contain a reference to a grey opacity at the back of the lens of the right eye, with very fine thread-like vessels radiating over the anterior part.

A fine thin vessel (the hyaloid artery) is evidently carrying blood. The column is seen extending from the upper temporal artery to the opacity at the back of lens.

$$
\text { R.V. }=1 / 60 . \quad \text { L.V. } \bar{c}+6.0 \text { D. sph. }=6 / 12 \text {. }
$$

A careful examination of all the details has been undertaken in the last few weeks, and the following conclusions have been arrived at.

The Iris.-This shows a fine strand of pupillary membrane extending obliquely on to the anterior capsule of the lens. Otherwise, there is no abnormality in this structure.

The Lens.-This shows a few small punctate opacities in the anterior cortex. Immediately behind the lens is the opacity in which the vessels are radiating. It is thicker centrally, thinning gradually to the periphery, and occupies about the middle third of the posterior part of lens. The opacity itself is thin, irregular and has a serrated margin. Through the centre a small opening can be seen, this is the point of entrance of the hyaloid artery, which divides into a descending branch and two nasal branches, the latter unite again into a common trunk. (See Fig. 1). With the slit-lamp, the blood is seen definitely coursing away from the centre, downwards in the descending branch, nasally in the other two branches. (See Fig. 2). The lower column can be traced as far as the posterior surface of the iris (the pupil being well dilated with atropine). The common trunk formed by the nasal vessels can be traced for a short distance and disappears behind the iris. In addition to these vessels, which are evidently carrying blood, two fine grey streaks, or strands, can be seen radiating from the central trunk laterally; one nearly at " 9 o'clock" and the other nearly at " 7 o'clock." (See Fig. 2). 


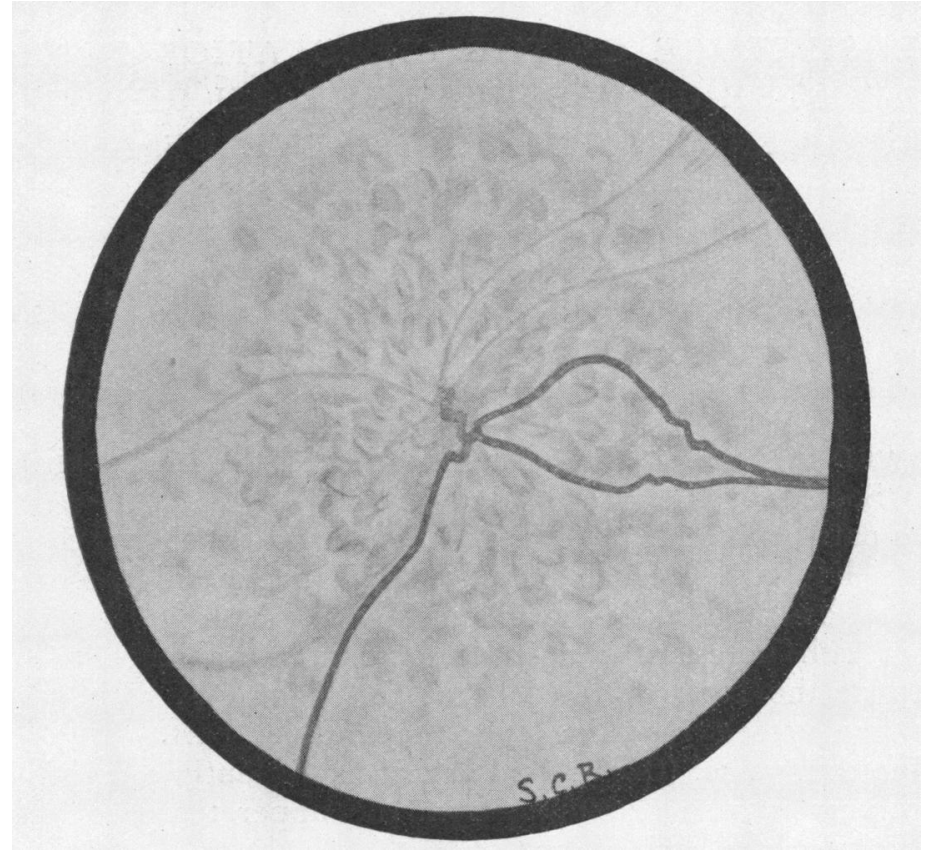

Fig. 2.-Silt-Lamp Appearance.

Some fine strands can be seen filling up the interval between " 12 o'clock and 3 o'clock." These, however, are not of the same definition as the two temporal ones described; they have the appearance of obliterated blood vessels.

The Hyaloid Artery. - Tracing the main trunk (which can be seen entering the opacity) backwards, we see two bends, or curves, which are made immediately behind the opacity; there is no irregularity of width, or bore, of this column, so far as I can make out. With the ophthalmoscope it appears black, but a red glint along the upper border can be obtained. The column is traced back to the disc. Here we experience the greatest difficulty in deciding its origin. The inferior temporal artery overlies the vein for a short distance, and it seemed to us that the vessel entered the artery just where it separates from the vein. We have finally come to the conclusion that the connective tissue overlying the vessels is responsible for this impression, and that the column ascends and enters the central artery. In other words, the hyaloid artery comes from the central artery and is involved in the connective tissue for part of its course from the disc. There is a certain amount of upward and downward movement of the column in the vitreous.

I am indebted to $\mathrm{Mr}$. Crawley for permission to publish this case, and to $\mathrm{Mr}$. Somerville Large for his help in studying it. 